\title{
Holographic storage using shift multiplexing
}

\author{
Demetri Psaltis, Michael Levene, Allen Pu, and George Barbastathis \\ Division of Engineering and Applied Science, California Institute of Technology, Pasadena, California 91125
}

Kevin Curtis

AT\&T Bell Laboratories, Room 1A346, 600 Mountain Avenue, Murray Hill, New Jersey 07974

Received September 26, 1994

\begin{abstract}
We demonstrate theoretically and experimentally a new multiplexing method for volume holographic storage using a single reference beam that is composed of multiple plane waves or is a spherical wave. We multiplex the holograms by shifting the recording material or the recording/readout head. The volume properties of the recording medium allow selective readout of holograms stored in successive overlapping locations. High storage densities can be achieved with a relatively simple implementation by use of the new method.
\end{abstract}

Volume holograms are usually recorded by use of angular,,$^{1,2}$ wavelength, ${ }^{3,4}$ phase code, ${ }^{5,6}$ and spatial ${ }^{7}$ multiplexing. In addition, peristrophic multiplexing, a holographic technique that applies to either thin or thick (three-dimensional) media, was recently introduced. ${ }^{8}$ Any of these methods or their combinations can be used to multiplex holograms for holographic storage devices.

In this Letter we introduce a method for multiplexing holograms by using a reference beam consisting of a spectrum of plane waves (similar to phase code multiplexing). We achieve multiplexing by shifting the recording medium with respect to the signal and reference beams. Alternatively, the two beams can be translated in tandem with respect to the stationary medium.

The geometry for shift multiplexing is shown in Fig. 1 for the case of storing Fourier transform holograms. The reference originates from an array of $M$ point sources located in the front focal plane of a Fourier lens and centered around the optical axis $z$. The lens transforms the field into a fan of $M$ plane waves. The angular separation is uniform, given by $\Delta \theta \approx d / F_{r}$, where $d$ is the distance between successive point sources and $F_{r}$ is the focal length. Thus, the angle of incidence of the $m$ th component is

$$
\theta_{m} \approx\left(m-\frac{M-1}{2}\right) \Delta \theta, \quad m=0, \ldots, M-1 .
$$

The angle of incidence of the central component of the signal with respect to the $z$ axis is denoted by $\theta_{S}$.

Because the reference consists of $M$ plane waves, we can think of the recording as consisting of $M$ separate holograms recorded simultaneously. On reconstruction, each plane wave in the reference fan reads out not only the hologram that it recorded but also all the holograms recorded by the other plane waves of the reference fan. These reconstructions, or ghosts, produce images that are shifted with respect to the primary reconstruction as a result of the change in readout angle relative to the recording angle. The ghosts are Bragg mismatched by an amount roughly proportional to the angular separation between the plane-wave component that originally recorded the hologram and the component that is reconstructing it. For the hologram recorded between the central signal component and the $m=0$ th reference component the amount of Bragg mismatch is $\Delta k_{z}=2 \pi l \tan \theta_{S} \Delta \theta / \lambda$ when read out by the $\pm l$ th reference component. The same relationship holds approximately for the other holograms. The diffraction efficiency of these Bragg-mismatched holograms is proportional to

$$
\eta(\Delta k)=\operatorname{sinc}^{2}\left(\frac{\Delta k_{z} L}{2 \pi}\right)
$$

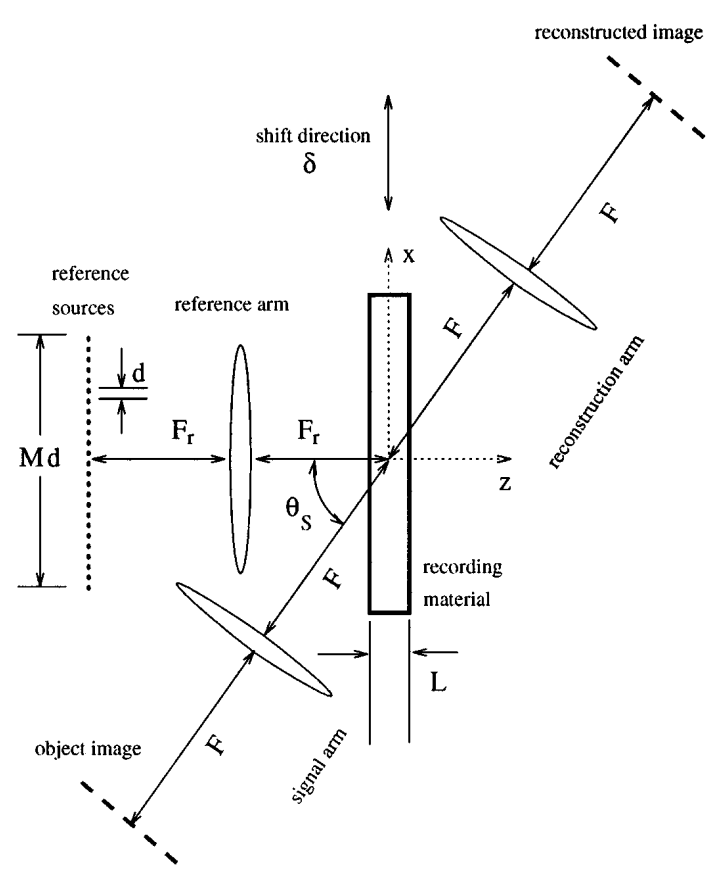

Fig. 1. Geometry for shift multiplexing in the Fourier plane. $F$ is the focal length of the Fourier transforming lens. 
where $\operatorname{sinc}(x)=\sin \pi x /(\pi x)$ and $L$ is the thickness of the recording medium. It follows that by choosing the angular separation $\Delta \theta$ between the reference components such that the sinc function of Eq. (2) vanishes, the ghosts will be eliminated, leaving a clean reconstruction. From Eq. (2) the required separation is

$$
\Delta \theta \approx \frac{\lambda}{L \tan \theta_{S}}
$$

Having eliminated the ghosts, we now examine what happens to the diffracted light if the hologram is shifted by a distance $\delta$ in the $x$ direction (see Fig. 1). We obtain the diffracted field $\mathcal{F}_{d}$ by multiplying the illuminating reference (consisting of $M$ plane waves) by the expression for the $M$ recorded holograms shifted by $\delta$. For a single plane-wave signal beam of incidence angle $\theta_{S}$ we have

$$
\begin{aligned}
\mathcal{E}_{d}= & \sum_{m} \exp \left(j 2 \pi \frac{m \Delta \theta x}{\lambda}\right) \sum_{m^{\prime}} \exp \left[-j 2 \pi \frac{m^{\prime} \Delta \theta(x-\delta)}{\lambda}\right] \\
& \times \exp \left(j 2 \pi \sin \theta_{S} \frac{x-\delta}{\lambda}\right), \\
\approx & {\left[\sum_{m} \exp \left(j 2 \pi \frac{m \Delta \theta \delta}{\lambda}\right)\right] \exp \left(j 2 \pi \sin \theta_{S} \frac{x-\delta}{\lambda}\right) . }
\end{aligned}
$$

The three-dimensional nature of the hologram (i.e., the $z$ dependence) serves to eliminate the cross terms $m \neq m^{\prime}$ (ghosts) from the double summation. When a signal with finite bandwidth is reconstructed, a detailed calculation (not given here) shows that the cross terms are not eliminated completely but the signal is still reconstructed with a high signal-tonoise ratio. From relation (5) the diffracted field consists of the reconstruction of the signal at angle $\theta_{S}$, weighted by a sum leading to the familiar array function. ${ }^{9}$ Therefore the intensity of the diffracted field as a function of shift is

$$
I(\delta) \propto \frac{\sin ^{2}\left(\pi M \frac{\delta \Delta \theta}{\lambda}\right)}{\sin ^{2}\left(\pi \frac{\delta \Delta \theta}{\lambda}\right)} .
$$

The zeros of the array function occur at

$$
\delta_{l}=l \frac{\lambda}{M \Delta \theta}, \quad l=1, \ldots, M-1 .
$$

Multiplexing is performed by recording each hologram with a shift $\delta_{1}=\lambda / M \Delta \theta$ with respect to its two neighbors. Because of the periodicity of the array function, at maximum $M$ holograms can be superimposed on the same location. The period is

$$
\delta_{M}=\frac{\lambda}{\Delta \theta} .
$$

The shift multiplexing method is particularly well suited for the implementation of holographic threedimensional disks. ${ }^{10}$ One can readily implement a three-dimensional disk with this method by simply using the disk rotation (which is already part of the system intended to permit accessing of information on different locations on the disk surface) to implement the shift (Fig. 2). This simplifies the design of the head because no additional components are required for selective readout.

The storage density $\mathcal{D}$ per unit area that we can achieve by using the device of Fig. 2 is limited by the thickness-dependent angular selectivity [relation (3)], the number of beams $M$ allowed by the optics, the page size $N_{p} b_{p}\left(b_{p}\right.$ is the pixel size and $N_{p}$ the number of pixels), and the periodicity of the array function. An approximate formula for the image plane density is

$$
\mathcal{D}=\frac{M \cos \theta_{S}}{b_{p}^{2}\left(1+M \delta_{1} \cos \theta_{S} / N_{p} b_{p}\right)}
$$

For $L=100 \mu \mathrm{m}$ and signal incidence angle $\theta_{S}=$ $30^{\circ}$, use of $F / 1$ optics permits $M=100$ holograms. Then, for typical page parameters $N_{p}=1000$ and $b_{p}=2 \mu \mathrm{m}$, Eq. (9) yields $\mathcal{D}=21.1 \mathrm{bits} / \mu \mathrm{m}^{2}$.

We demonstrated array multiplexing using a reference fan of 20 plane waves angularly separated by $0.5^{\circ}$. The recording material was DuPont HRF-150 polymer of thickness $L=38 \mu \mathrm{m}$. The effect of shift on the reconstruction of a single hologram is shown in Fig. 3. The signal image was a $100 \times 100$ random

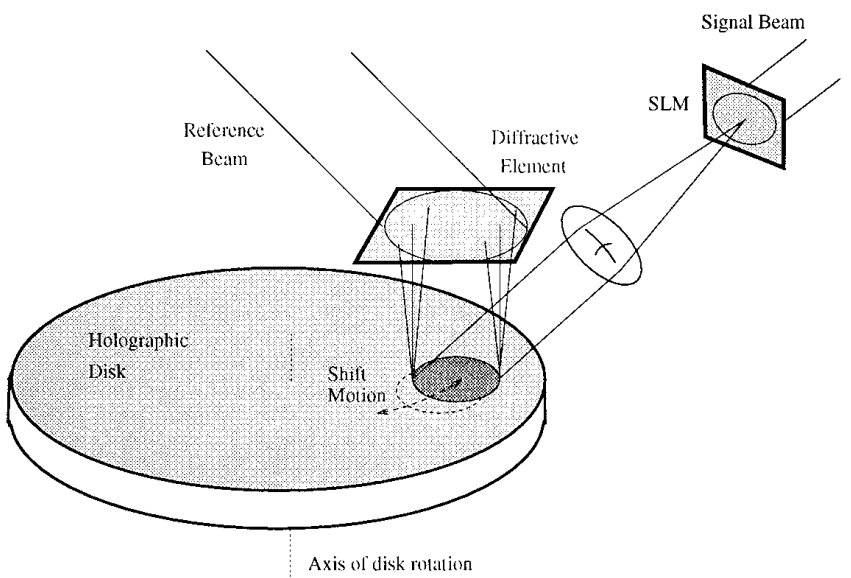

Fig. 2. Holographic three-dimensional disk with shift multiplexing setup. SLM, spatial light modulator.

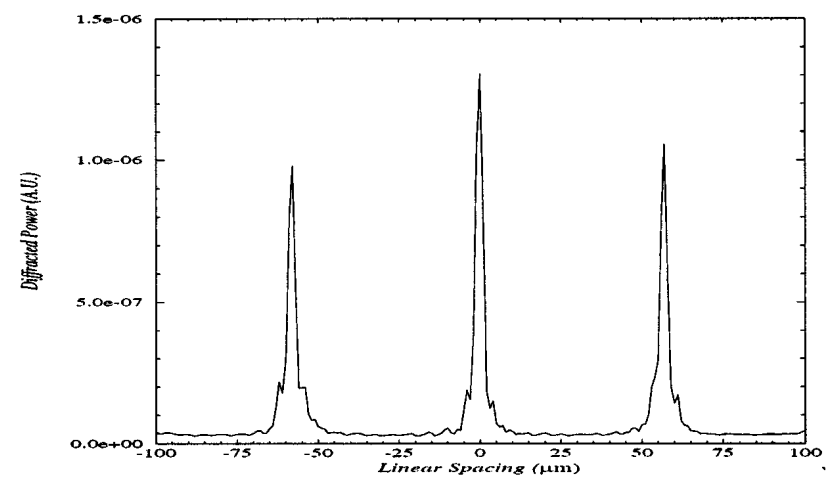

Fig. 3. Experimental demonstration of the array function with a single hologram of a random bit pattern. 


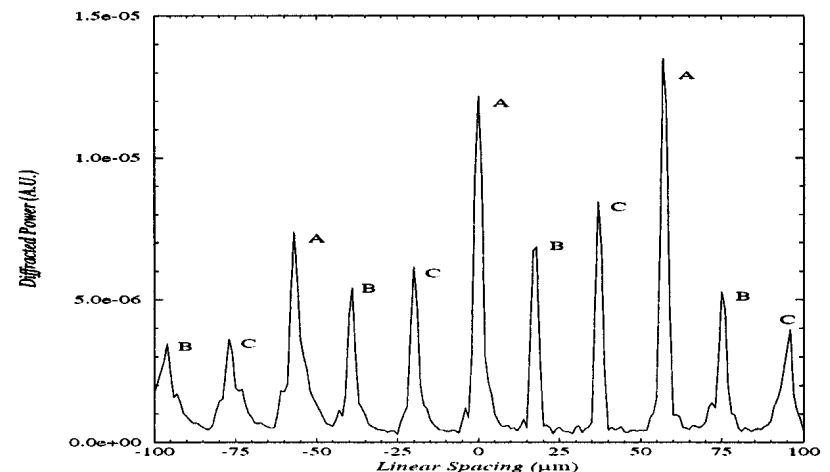

Fig. 4. Multiplexing of three holograms (A, B, C) of random bit patterns by the shift method.

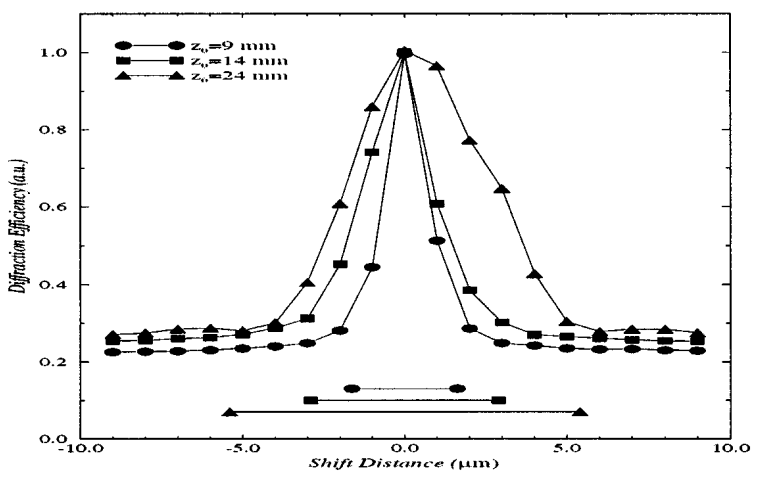

Fig. 5. Experimental shift selectivity of a volume hologram recorded with a spherical reference wave. The bars indicate the theoretical prediction.

bit pattern. For the above parameters the theoretical shift selectivity is $2.8 \mu \mathrm{m}$ and the period is $55 \mu \mathrm{m}$, in good agreement with the experiment. The reason for the deviation from the theoretically predicted periodicity is the finite transverse size of the recording region.

Three holograms array multiplexed with the same setup are shown in Fig. 4. Each hologram is reconstructed almost periodically, following its own array function. Because of the very small thickness of the recording medium in this experiment, we used an angular separation smaller than that predicted by Eq. (3). Therefore the ghosts had to be filtered out in the Fourier plane.

Shift multiplexing can also be implemented by a spherical wave reference ${ }^{11}$ instead of the fan of $M$ plane waves. The basic operation is similar, but the analysis is more involved. Consider a spherical reference wave originating a distance $z_{0}$ from the center of the recording material and a plane-wave signal incident at angle $\theta_{S}$ with respect to the optical axis. An approximate calculation (under the paraxial and Born approximations and neglecting variable modulation depth) predicts that the shift selectivity is re- lated to the focal distance and the Bragg angular selectivity [relation (3)] as $\delta_{\text {Bragg }} \approx z_{0} \Delta \theta=\lambda z_{0} / L \tan \theta_{S}$. The finite numerical aperture (NA) broadens the selectivity curve by a factor of $\delta_{\mathrm{NA}} \approx \lambda / 2(\mathrm{NA})$. Therefore we have

$$
\delta_{\text {spherical }}=\delta_{\text {Bragg }}+\delta_{\mathrm{NA}} \approx \frac{\lambda z_{0}}{L \tan \theta_{S}}+\frac{\lambda}{2(\mathrm{NA})}
$$

Figure 5 shows the experimental shift selectivity curves for $z_{0}=9,14$, and $24 \mathrm{~mm}$ and NA's of $0.3,0.15$, and 0.075 , respectively. The angle of incidence of the signal beam was $40^{\circ}$ outside the 8-mm-thick iron-doped $\mathrm{LiNbO}_{3}$ crystal (refractive in$\operatorname{dex} n \approx 2.24)$. The experimental selectivity agrees with relation (10).

In conclusion, we have demonstrated shift multiplexing in photopolymers and photorefractives. The new method is promising for the achievement of high holographic storage density with a relatively simple implementation.

We thank Hsin-Yu Sidney Li, Geoffrey W. Burr, and Fai H. Mok for helpful discussions. This research was supported by the U.S. Air Force Office of Scientific Research. Michael Levene is supported by a National Defense Science and Engineering Graduate Fellowship. George Barbastathis is grateful to the Charles Lee Powel Foundation for supporting his first year at Caltech through a Graduate Fellowship.

\section{References}

1. D. L. Staebler, J. J. Amodei, and W. Philips, in Digest of Seventh International Quantum Electronics Conference (Joint Council on Quantum Electronics, Montreal, 1972), p. 611.

2. F. H. Mok, Opt. Lett. 18, 915 (1993).

3. G. A. Rakuljic, V. Levya, and A. Yariv, Opt. Lett. 17, 1471 (1992).

4. S. Yin, H. Zhou, F. Zhao, M. Wen, Y. Zang, J. Zhang, and F. T. S. Yu, Opt. Commun. 101, 317 (1993).

5. C. Denz, G. Pauliat, and G. Roosen, Opt. Commun. 85, 171 (1991).

6. J. E. Ford, Y. Fainman, and S. H. Lee, Opt. Lett. 15, 1088 (1990).

7. F. H. Mok, G. W. Burr, and D. Psaltis, Opt. Mem. Neural Net. 3, 119 (1994).

8. K. Curtis, A. Pu, and D. Psaltis, Opt. Lett. 19, 993 (1994).

9. J. D. Kraus, Antennas (McGraw-Hill, New York, 1950), pp. 74-79.

10. H.-Y. S. Li and D. Psaltis, Appl. Opt. 33, 3764 (1994).

11. L. Solymar and D. J. Cooke, Volume Holography and Volume Gratings (Academic, New York, 1981), pp. 243-253 and references therein. 